\title{
ARTICLE Intestinal dysbiosis compromises alveolar macrophage immunity to Mycobacterium tuberculosis
}

\author{
Nargis Khan ${ }^{1}$, Laura Mendonca ${ }^{1}$, Achal Dhariwal ${ }^{2}$, Ghislaine Fontes ${ }^{3}$, Dick Menzies ${ }^{4}$, Jianguo Xia ${ }^{2,3,5}$, Maziar Divangahi ${ }^{1,3,4}$ and $^{2}$ \\ Irah L. King ${ }^{1,3}$
}

\begin{abstract}
Current treatments for tuberculosis (TB) are effective in controlling Mycobacterium tuberculosis (Mtb) growth, yet have significant side effects and do not prevent reinfection. Therefore, it is critical to understand why our host defense system is unable to generate permanent immunity to $M t b$ despite prolonged anti-tuberculosis therapy (ATT). Here, we demonstrate that treatment of mice with the most widely used anti-TB drugs, rifampicin (RIF) or isoniazid (INH) and pyrazinamide (PYZ), significantly altered the composition of the gut microbiota. Unexpectedly, treatment of mice with the pro-Mtb drugs INH and PYZ, but not RIF, prior to Mtb infection resulted in an increased bacterial burden, an effect that was reversible by fecal transplantation from untreated animals. Mechanistically, susceptibility of INH/PYZ-treated mice was associated with impaired metabolism of alveolar macrophages and defective bactericidal activity. Collectively, these data indicate that dysbiosis induced by ATT administered to millions of individuals worldwide may have adverse effects on the anti-Mtb response of alveolar macrophages.
\end{abstract}

Mucosal Immunology (2019) 12:772-783; https://doi.org/10.1038/s41385-019-0147-3

\section{INTRODUCTION}

Mycobacterium tuberculosis (Mtb) has co-evolved with humans to achieve an evolutionary trade-off that leads to prolonged survival within the host respiratory system. Although much is known about the chronic stages of disease, little is understood about the early phase of infection when $M t b$ is still in the airway and has yet to translocate into the lung parenchymal tissue. Considering that alveolar macrophages constitute $>90 \%$ of the leukocytes in the airways of naive hosts, their bactericidal capacity is central to early immunity to $M t b$ infection. Thus, it is not surprising that $M t b$ has evolved mechanisms to infect and survive within alveolar macrophages. Conversely, epidemiological studies indicate that among close household contacts of highly active TB patients, $50 \%$ of exposed individuals are completely resistant to TB and are tuberculin skin test-negative, indicating that $M t b$ can be eliminated by a robust innate immune response. ${ }^{1,2}$ Therefore, maximizing the functional capacity of alveolar macrophages during early Mtb infection is critical for preventing TB.

Current regimens to control TB rely on protracted use of antituberculosis therapy (ATT) including 4-9 months of daily treatment with single or multidrug combinations. ${ }^{3}$ Although these treatments are efficient at killing $M t b$, they are strongly associated with side-effects including liver toxicity ${ }^{4}$ as well as poor compliance, and the development of multidrug-resistant strains. ${ }^{5}$ Further, current treatment strategies do not consider their impact on reinfection or reactivation of disease, which also significantly contributes to the global incidence of TB. ${ }^{6}$ For example, a study conducted in Cape Town, South Africa indicated that individuals receiving ATT are seven times more likely to develop active TB compared with untreated individuals. ${ }^{7}$ These paradoxical effects of ATT provide the impetus for investigating the systemic and potentially untoward effects of these drugs on the host response to Mtb infection.

Recent studies of the gut microbiome have demonstrated that alterations in the intestinal microbial ecosystem (i.e., dysbiosis) are associated with an increased risk of numerous metabolic and autoimmune diseases, including obesity, atopic dermatitis, and diabetes. $^{8}$ Moreover, substantial evidence indicates that antibiotic-induced dysbiosis promotes susceptibility to infectious diseases. Broad-spectrum antibiotic administration to mice increased susceptibility to intestinal $L$. monocytogenes and Salmonella serovar Typhimurium infections. ${ }^{9-11}$ Intestinal dysbiosis not only impacts enteric infection, but can also compromise immunity at other barrier sites including the respiratory tract. For example, broad-spectrum antibiotic treatment compromised anti-viral immunity following influenza infection, and was associated with decreased TNFa and IFN $y$ production by CD8 + T cells. ${ }^{12}$ In addition, we have recently shown that a similar broad-spectrum antibiotic regimen compromises immunity to pulmonary Mtb infection. ${ }^{13}$ However, the direct impact of antiTB antibiotics-administered to millions of individuals living with latent or active TB-on the gut microbiome and subsequent susceptibility to $M t b$ infection has not been investigated.

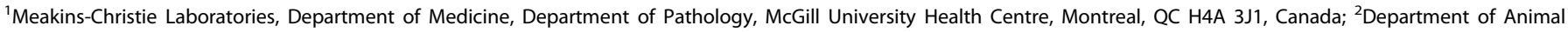

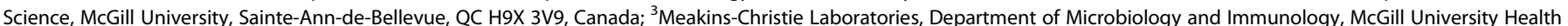

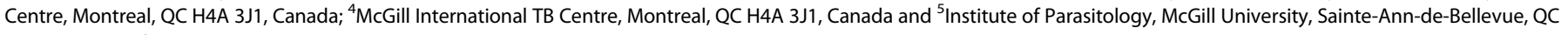
H9X 3V9, Canada

Correspondence: Maziar Divangahi (maziar.divangahi@mcgill.ca) or Irah L. King (irah.king@mcgill.ca)

These authors contributed equally: Laura Mendonca, Achal Dhariwal, Maziar Divangahi, and Irah L. King
}

Received: 10 April 2018 Revised: 27 January 2019 Accepted: 29 January 2019

Published online: 19 February 2019 
The most commonly used anti-TB drugs include isoniazid (INH), pyrazinamide (PYZ), and rifampicin (RIF). INH and PYZ are pro-drugs that are commonly administered together and are thought to specifically target the mycobacterial cell wall, whereas RIF, a bacterial RNA polymerase inhibitor, has broadspectrum activity against gram-positive and gram-negative bacteria. ${ }^{14}$ Indeed, recent studies have shown that ATT following $M t b$ infection in both mice and humans induced gut dysbiosis. ${ }^{15,16}$ However, the functional impact of this dybiosis is still unknown.

In this study, we hypothesized that ATT-induced changes to the gut microbiome promotes susceptibility to pulmonary $M t b$ infection. Our results demonstrate that the anti-TB antibiotics INH and PYZ, but not RIF, had unique effects on the gut microbiome that increased susceptibility to Mtb infection. Importantly, the effects on bacterial burden were reversible with fecal transplantation from naive mice. Interestingly, the ATT-dependent increase in susceptibility to Mtb infection was associated with an altered metabolic profile of alveolar macrophages that compromised their bactericidal capacity.

\section{RESULTS}

ATT alters the gut microbiome

Recent studies have demonstrated that ATT changes the composition and diversity of the mouse and human microbiome. $^{15,16}$ To confirm these observations, C57BL/6 mice were treated with either the pro-drugs INH and PYZ or the broad-spectrum antibiotic RIF in the drinking water. Fecal pellets were collected at different time points prior to and after the treatments and subjected to sequencing of the V3 hypervariable region from the bacterial $16 \mathrm{~S}$ rDNA locus. Although the baseline microbiome compositions before treatments were similar between animal cages at both phylum and family levels (Supplementary Figure 1A, B), significant changeswere observed in both microbial composition and diversity beginning at day 2 of drug treatments (Supplementary Figure 1C). As TB drug-induced changes to the gut microbiota are stable by 12 days post treatment, this time point was used for further analysis. ${ }^{15}$ Consistent with a previous report, ${ }^{16}$ RIF treatment led to significant operational taxonomic unit (OTU) changes in both within-sample (alpha) diversity and between-sample (beta) diversity, as determined by Chao1 indices and unweighted UniFrac analyses, respectively (Fig. 1a-c). By contrast, INH/PYZ treatment had only modest effects as shown in both OTU alpha and beta diversity profiles (Fig. 1b-d).

The gut microbiota is primarily composed of five phyla including Bacteroidetes, Firmicutes, Actinobacteria, Proteobacteria, and Verrucomicrobia. RIF treatment led to an expansion of Bacteroidetes and Verrucomicrobia phyla with a concomitant decrease in Firmicutes (Fig. 1e). Unexpectedly, treatment withINH/PYZ, previously thought to be specific for mycobacterial species, also increased the representation of Bacteroidetes (Fig. 1e). Similar changes were observed at the family level with expansion of Bacteroides, Verrucomicrobiaceae, and a decrease in Lachnospiraceae in RIF-treated samples, whereas INH/PYZ-treated mice showed an increase of Clostridiaceae only (Fig. 1f). Differential abundance analysis was performed using the metagenomeSeq method. ${ }^{17}$ Significant genus-level features were also observed following RIF or INH/PYZ treatment (Fig. 1g, h; Supplementary Figure 1D and E). For example, the most significant changes in the INH/PYZ-treated group were observed in the Clostridia IV and XIV clusters, whereas the changes observed in the RIF-treated group were much more diverse. These data indicate that both RIF and INH/PYZ ATT significantly and uniquely alter the gut microbiome.
INH/PYZ-driven changes to the gut microbiome are associated with increased burden and dissemination of $M t b$

To determine whether the observed ATT-induced changes to the gut microbiota had an impact on host resistance to $M t b$ infection, mice were treated with RIF or INH/PYZ in the drinking water prior to $M t b$ challenge. After 8 weeks, treatment was stopped and, 2 days later, mice were infected with virulent $M t b$ via the aerosol route (Fig. 2a). Five weeks after Mtb infection, bacterial burden in the lungs and spleen of antibiotic-treated mice were compared to untreated animals. Animals pre-treated with INH/PYZ harbored a significantly greater bacterial burden in the both the lungs and spleen compared with untreated controls (Fig. 2b, c). However, no changes were observed in the bacterial burden of RIF-treated mice (Fig. 2d, e). As TB patients frequently receive both RIF and INH/PYZ (RHZ) treatment, we tested whether combined ATT would have an additive or synergistic effect on susceptibility to Mtb. However, the $M t b$ burden in mice after combined treatment was similar to INH/ PYZ treatment alone (Fig. 2f, g). These results indicate that INH/ PYZ, but not RIF, pre-treatment compromises host defense against Mtb infection.

Though we observed an increase in Mtb burden in mice pretreated with INH/PYZ, whether these changes were owing to gut microbiota alterations was not clear. To test this possibility, mice were pre-treated with RIF or INH/PYZ and then were fed with fecal preparations collected from naive mice prior to $M t b$ infection (Fig. 2a). Notably, INH/PYZ-treated mice showed a significantly reduced $M t b$ burden, to the level of untreated infected animals, in the lungs and a similar trend was observed in spleen after fecal transplantation (Fig. 2b, c), whereas fecal transplantation in RIFtreated mice had no effect on Mtb burden in either organ (Fig. 2d, e).

To determine whether the changes in bacterial burden following fecal transplantation was associated with a reconstitution of the gut microbiota, we performed quantitative real-time polymerase chain reaction (qRT-PCR) analysis for Clostridium XIV bacteria (Firmicutes phyla), the cluster most affected by INH/PYZ treatment, after the last fecal transplant. Consistent with our sequencing results, we observed a significant increase in Clostridium XIV bacteria in the INH/PYZ-treated mice that could largely be restored to baseline after fecal transplant (Fig. 2h). Collectively, these observations indicate that INH/PYZ-specific changes to the gut microbiota compromise host resistance to Mtb infection.

ATT-driven changes to the gut microbiome does not impact the Mtb-specific T-cell response

$\mathrm{T}$ cells are considered a cornerstone of protection against $M t b$ infection. Therefore, we next investigated whether failure of $\mathrm{INH} / \mathrm{PYZ}$-treated mice to control $M t b$ growth was owing to defective expansion of antigen-specific T cells. To this end, mice were treated with INH/PYZ for 2 months and then challenged with aerosolized $M t b$. Five weeks after infection, no difference in the frequency of tetramer-positive TB10.4-specific CD8 + T cells or ESAT-6-specific CD4 T+ cells were observed in the lungs of INH/PYZ-treated mice compared with controls (Fig. 3a-d). Similarly, no difference was observed following RIF treatment or upon fecal transplantation (Fig. 3e). As Th1 cells are the main effector CD4 T cells for mediating protection against $M t b,{ }^{18}$ we examined expression of the Th1 lineage-specific transcription factor, T-bet. There was no significant change in the frequency of T-bet + CD4 T cells in the lungs of either INH/PYZ or RIFtreated infected mice compared with controls (Fig. 3f). Regulatory CD4 $+T$ cells (Treg) also determine the susceptibility of the host to Mtb infection by delaying the arrival of Th1 cells to site of infection. ${ }^{19}$ In addition, the gut microbiota has a potent influence on the generation of regulatory $T$ cells. ${ }^{20}$ However, neither RIF-treated nor INH/PYZ-treated mice showed any difference in the frequency of pulmonary Treg cells compared with untreated mice (Fig. 3g). 


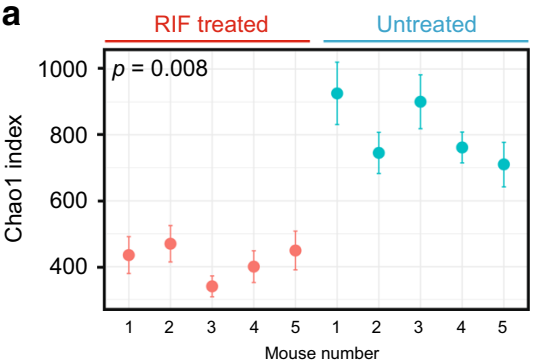

C

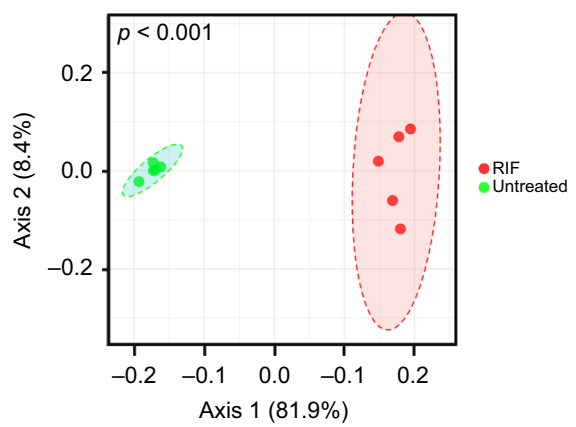

e

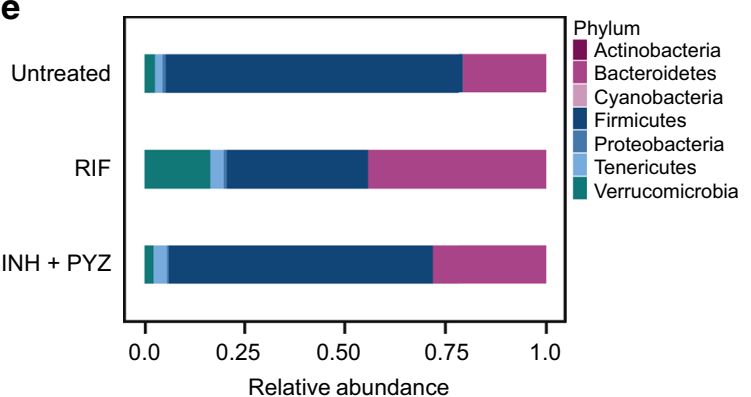

g

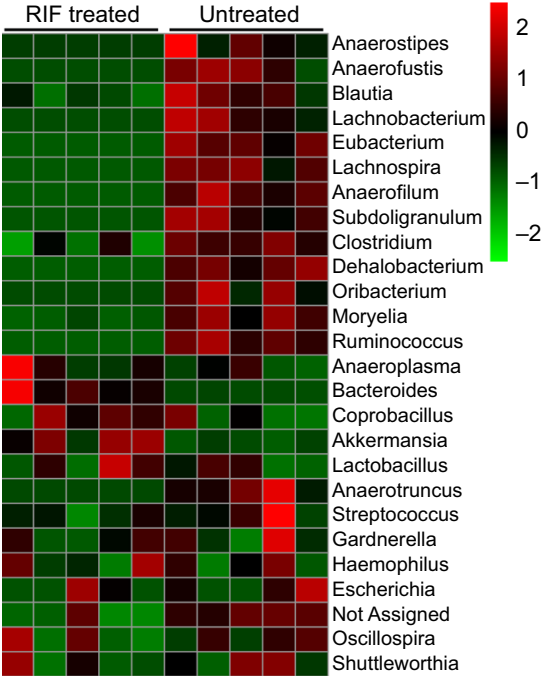

b

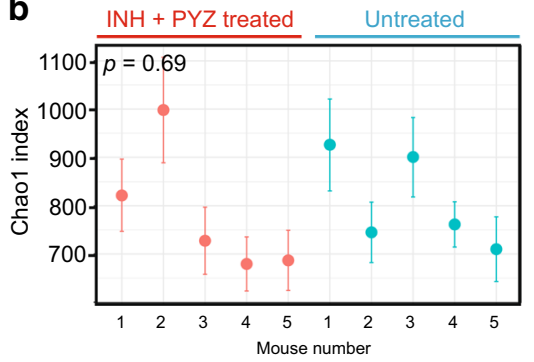

d

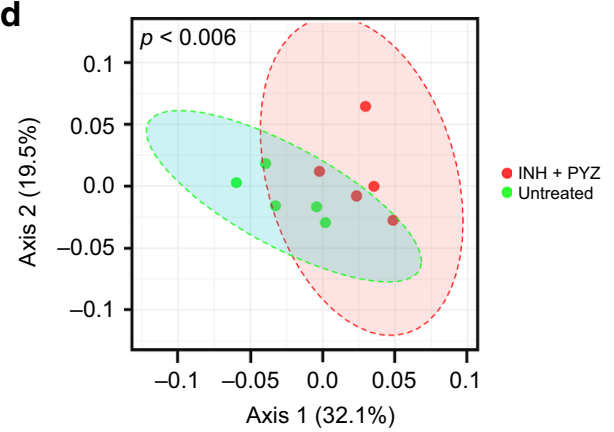

f

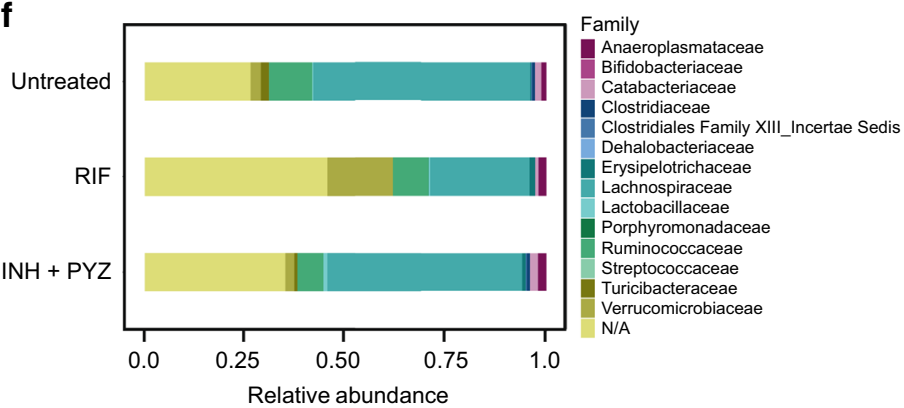

h

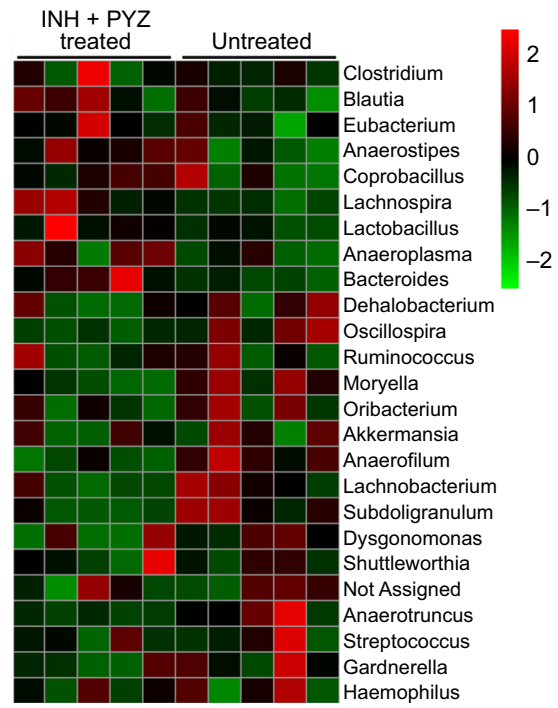

Fig. 1 ATT alters the gut microbiome. Mice were treated with RIF or INH/PYZ for 12d. Shift in the microbiota composition from untreated samples was calculated from the sequenced data and expressed as a, $\mathbf{b}$. Alpha diversity using Chao1 (RIF, $p=0.0079365 ; \mathrm{INH}, p=0.69048)$. c, d Beta diversity using unweighted UniFrac distances (RIF, $p<0.001$; INH, $p<0.006$ ). e, f Bar chart representing the composition at the phylum and family levels after $12 \mathrm{~d}$ of treatment. $\mathbf{g}$, $\mathbf{h}$ Heat map comparison between the untreated group and after RIF or INH/PYZ treatment at the genus level

Impaired innate response in INH/PYZ-treated mice Considering T-cell-mediated immunity was not impaired following ATT, we investigated whether the innate immune response during
Mtb infection was disrupted in mice that received $\mathrm{INH} / \mathrm{PYZ}$ treatment. First, we assessed the number of innate immune subsets in the lungs of INH/PYZ-treated mice 5 days post $M t b$ 
a

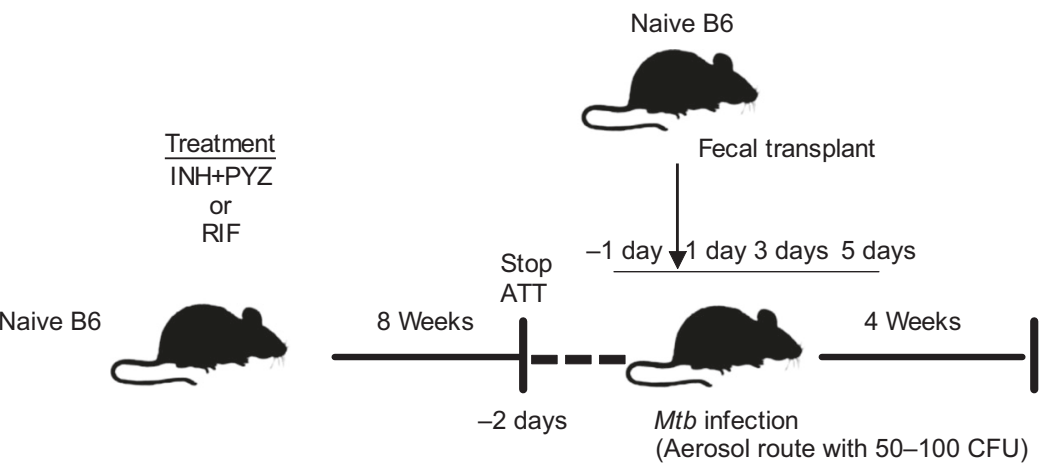

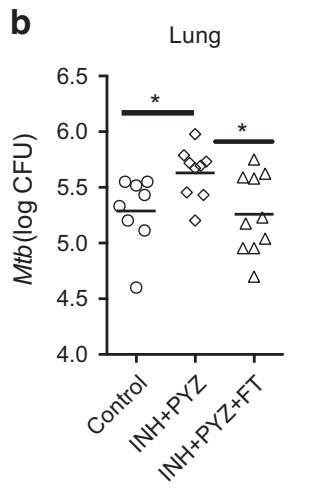

c
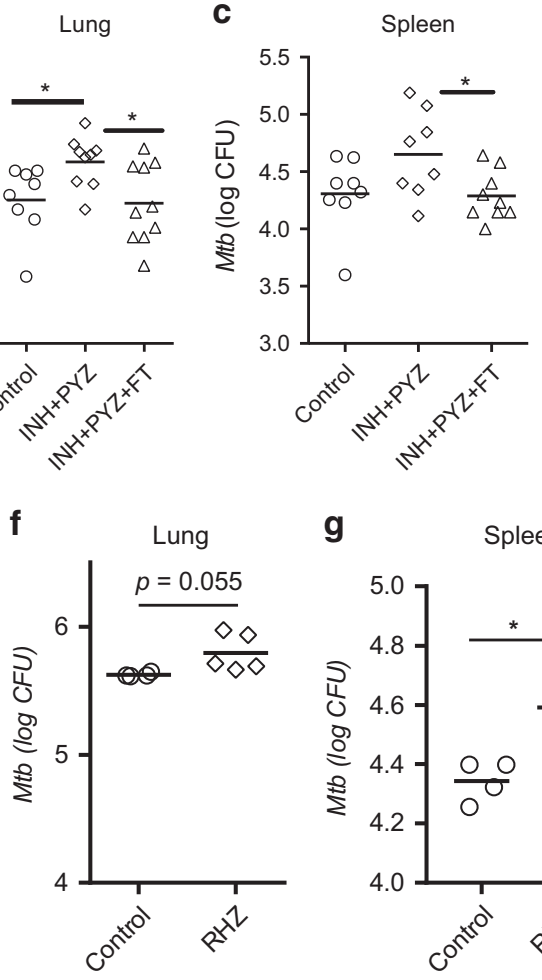

g Spleen

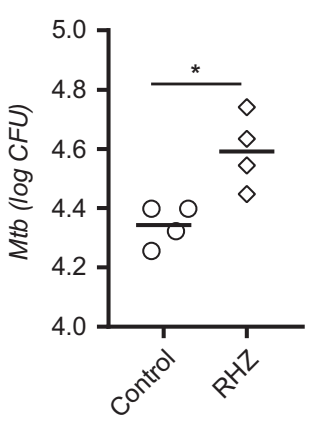

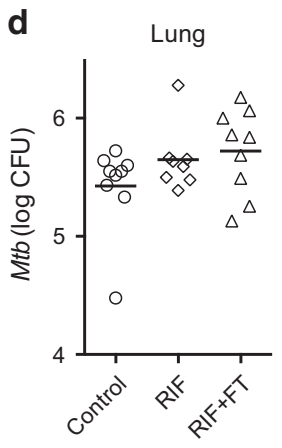

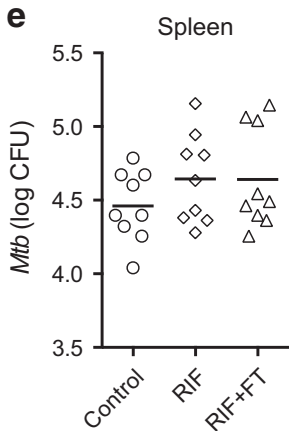

Fig. 2 Mice pre-treated with INH/PYZ show higher susceptibility to Mycobacterium tuberculosis infection. a Schematic representation for ATT and $M t b$ infection. Mice were treated with RIF or INH/PYZ for 8 wks. Treatment was stopped 2 days prior to Mtb infection. One group of treated mice received faeces collected from naive mice at $-1 d,+1 d,+3 d,+5 d$ of $M t b$ infection $(n=5 / g r o u p)$. Bacterial burden in lungs and spleen of $\mathbf{b}, \mathbf{c}$ INH/PYZ and INH + PYZ + FT; d, e RIF and RIF + FT-treated mice. $\mathbf{f}$, $\mathbf{g}$ Bacterial burden in lungs and spleen of mice treated with RIF/INH/ PYZ (RHZ) as described in $\mathbf{a}$. Data shown are pooled from two $\mathbf{b}-\mathbf{e}$ and one $\mathbf{f}$ and $\mathbf{g}$ independent experiments. $\mathbf{h}$ Relative abundance of Clostridia cluster XIV bacteria ( $n=4-5 /$ group). Data shown are representative of two independent experiments. Error bars represent mean \pm SEM; ${ }^{*} p<0.05$

infection. There was no change in the frequency or total cell counts of monocytes, interstitial macrophages, alveolar macrophages, eosinophils, or neutrophils, in the lungs of INH/PYZtreated mice compared with control mice (Fig. 4a, b). To broadly examine the activation status of the pulmonary mononuclear phagocyte compartment following antibiotic treatment, we assessed expression of CD80 and MHCII. Although no differences were observed in CD80 expression (unpublished observations), alveolar macrophages isolated from INH/PYZ-treated mice showed a selective and significant decrease in the expression of $\mathrm{MHCll}$ (Fig. 4c), whereas no differences in the expression of MHCII was observed on other innate immune cells, including monocytes and interstitial macrophages (Fig. 4d).

The dynamic cellular metabolism of macrophages reflects their antibacterial capacity. To assess the macrophage bioenergetic profile, we tested the oxygen consumption rate (OCR) to quantify mitochondrial respiration and extracellular acidification rate (ECAR) as an indicator of glycolysis. Alveolar macrophages from INH/PYZ-treated mice exhibited an altered metabolic profile with a significant reduction in basal respiration, maximal spare respiratory capacity and ATP production (Fig. $4 \mathrm{e}, \mathrm{f}$ ), but no change in ECAR (Fig. 4g). Previous evidence indicates that mitochondria dysfunction is associated with a defect in pro-inflammatory cytokines, including IL-1 $\beta{ }^{21}$ Similarly, we also found that the expression and secretion of two key anti-mycobacterial cytokines (TNFa and IL-1 $\beta$ ) were significantly reduced in alveolar macrophages from INH/PYZ-treated mice infected with Mtb (Fig. 4h, i).

Bactericidal and bacteriostatic antibiotics have also been shown to target mitochondrial components. ${ }^{22}$ To exclude the possibility that INH/PYZ treatment was directly affecting the function of alveolar macrophages, we isolated these cells from naive mice and treated them with INH/PYZ for two hours prior to an overnight rest 


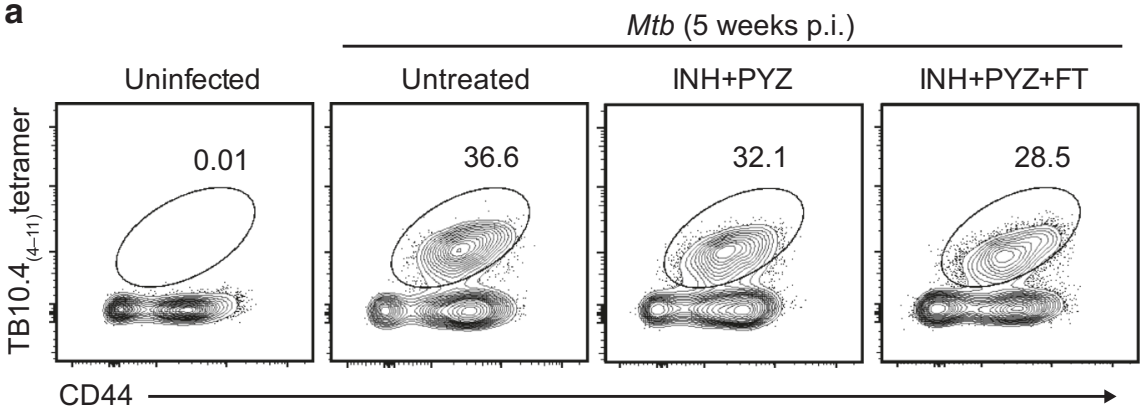

C

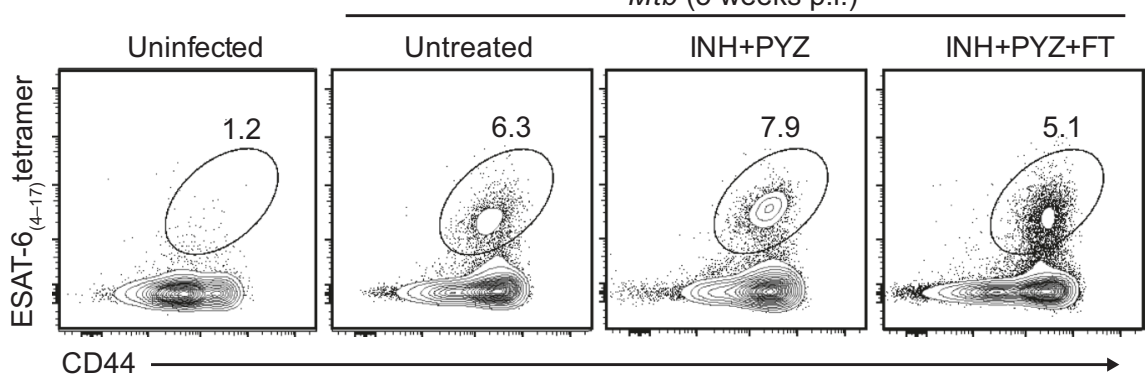

b

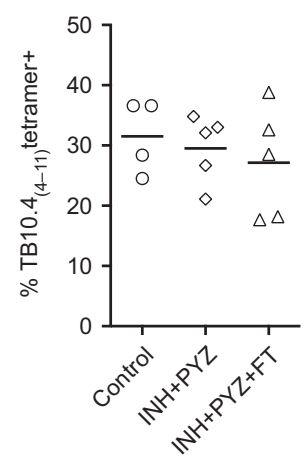

d

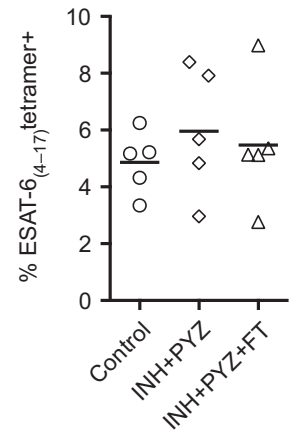

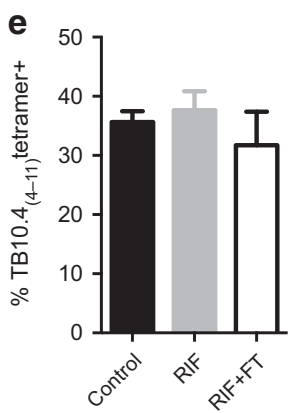
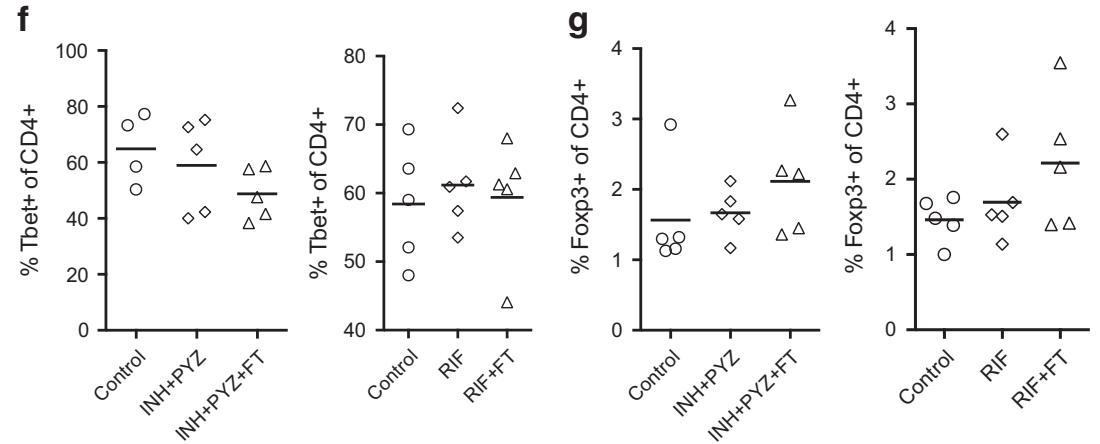

Fig. 3 Mycobacterium tuberculosis-infected mice pre-treated with INH/PYZ show no differences in antigen-specific T-cell responses. a-g Mice were treated with RIF or INH/PYZ for 8 wks. Treatment was stopped 2 days prior to Mtb infection. One group of treated mice received faeces collected from naive mice at $-1 d,+1 d,+3 d,+5 d$ of Mtb infection ( $n=5 /$ group). $\mathbf{a}, \mathbf{b}$, and e Frequency of TB10.4 $4-11)$ specific CD8 + T cells and $\mathbf{c}$, d ESAT- $6_{(4-17)}$-specific CD4 T + cells. $\mathbf{f}$ T-bet + or $\mathbf{g}$ FoxP3 + CD4 T cells in the lungs ( $n=5 / g r o u p$ ) at 4 weeks post infection with $M$ tb. Data shown are representative of two independent experiments. Error bars represent mean $\pm \mathrm{SEM}$

period as described above. In contrast to our results following in vivo treatment with $\mathrm{INH} / \mathrm{PYZ}$, in vitro treatment of alveolar macrophages had no effect on mitochondrial respiration (Fig. 4j, k).

Alveolar macrophages from INH/PYZ-treated mice fail to restrict the growth of $M t b$

Considering the importance of alveolar macrophages in controlling $M t b$ growth and their defective phenotype after INH/PYZ treatment in vivo, we harvested alveolar macrophages from mice treated with INH/PYZ or RIF and infected them with $M t b$ in vitro. Alveolar macrophages from untreated or treated mice showed similar levels of infection at $4 \mathrm{~h}$ post challenge indicating that ATT in vivo did not impact uptake of $M t b$ (Fig. 5a, b). However, INH/PYZ-treated macrophages failed to control Mtb growth as observed by an increased number of $M t b$ at day 3 post infection (Fig. 5a). In corroboration with our in vivo data, we did not observe a change in the bacterial load of alveolar macrophages from RIF-treated mice (Fig. 5b). As recruited monocyte-derived macrophages also restrict $M t b$ growth, $^{23,24}$ we differentiated bone marrow-derived macrophages (BMDM) from INH/PYZ or RIF-treated mice and then infected them with $M t b$. In contrast to alveolar macrophages, BMDM from antibiotictreated mice showed no difference in controlling Mtb growth (Fig. 5c).

To test whether the failure of alveolar macrophages to constrain the growth of $M t b$ was due to any direct effects of ATT, we harvested alveolar macrophages from naive mice and treated them with RIF or INH/PYZ for $2 \mathrm{~h}$. The cells were washed and incubated in fresh media overnight as in the experiments described above. Alveolar macrophages were then infected with $\mathrm{Mtb}$ ( $\mathrm{MOI}$ of 1 ) and bacterial burden was assessed at 4 and $72 \mathrm{~h}$. Irrespective of treatment, no differences in bacterial burden were 

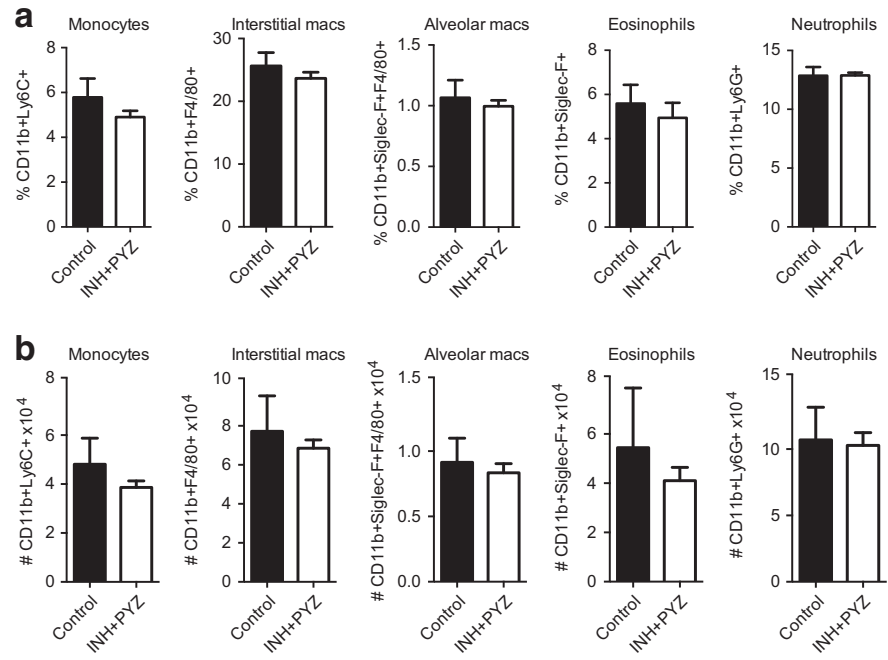

C
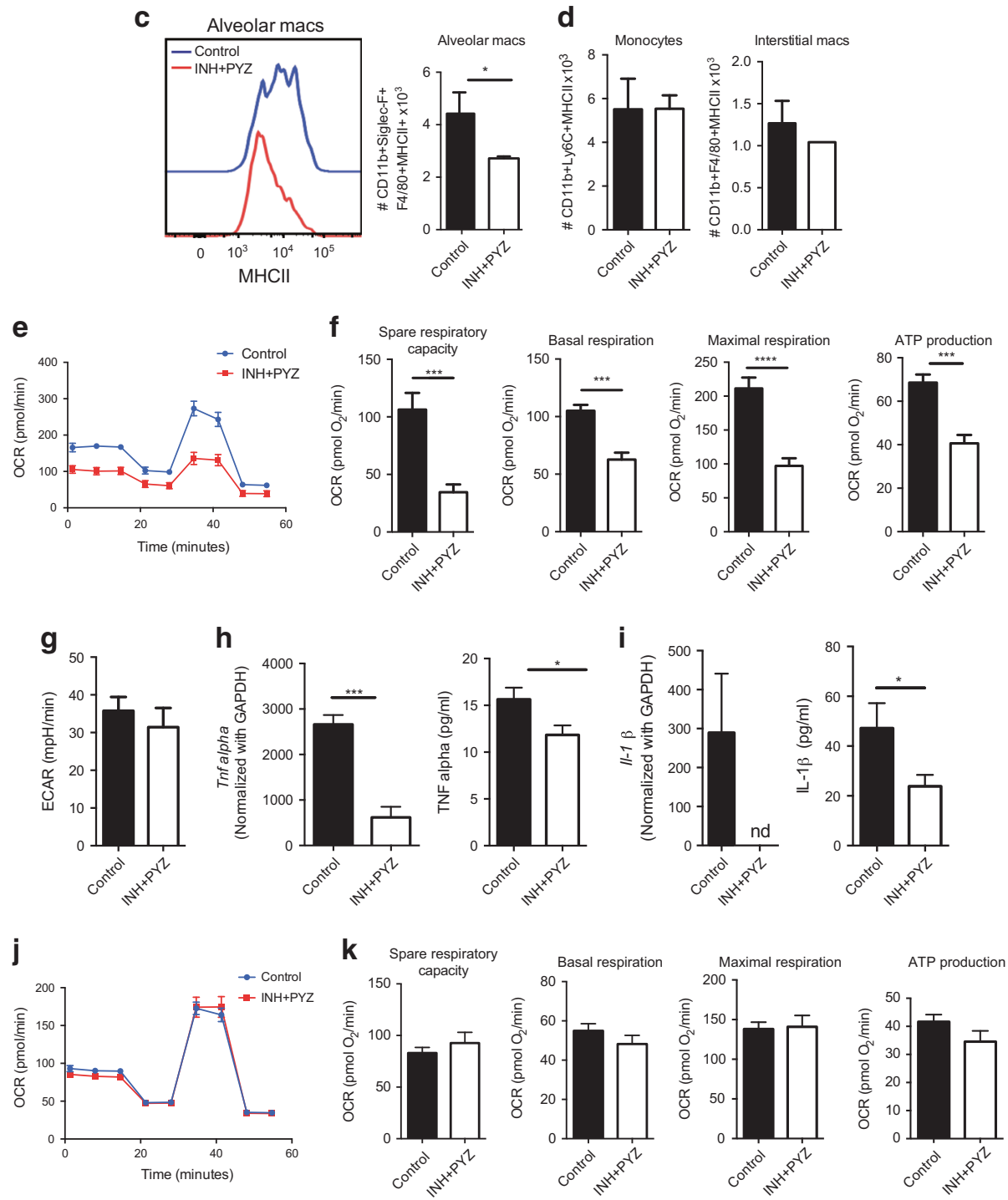

observed compared with controls (Fig. 5d). Collectively, our results indicate that the defect in alveolar macrophage bactericidal activity results from $\mathrm{INH} / \mathrm{PYZ}$-dependent changes to gut microbiota.

To further validate our observations in vivo, alveolar macrophages were harvested from control or INH/PYZ-treated mice and infected with $M t b$ in vitro prior to being adoptively transferred (intratracheally) into Rag1-/- mice (Fig. 5e). Rag1-/- mice were 
Fig. 4 ATT impairs the innate immune response. Mice were treated with RIF or INH/PYZ for 8 wks. Treatment was stopped 2 days prior to Mtb. After $5 \mathrm{~d}$ post infection, single-cell suspensions of lung cells were immunophenotyped by flow cytometry. $\mathbf{a}$ Frequency and $\mathbf{b}$ total cell number of pulmonary monocytes, interstitial macrophages, alveolar macrophages, eosinophils, and neutrophils as determined by the lineage-specific markers described on the $y$ axis of each graph. $\mathbf{c}$, d Expression of $\mathrm{MHCll}$ on $\mathbf{c}$ alveolar macrophages and $\mathbf{d}$ monocytes and interstitial macrophages. e-g Alveolar macrophages were harvested from INH/PYZ-treated mice and, using a Seahorse analyser, mitochondrial function was analysed through the addition of mitochondrial inhibitors in the following order: oligomycin A (1.5 $\mu \mathrm{m})$, carbonyl cyanide-4(trifluoromethoxy) phenylhydrazone (FCCP) $(2 \mu \mathrm{M})$, and antimycin A $(2 \mu \mathrm{M})$ as a function of time. The oxygen consumption (OCR), basal respiration, maximal respiration, spare respiratory capacity and ATP production was quantified in $\mathbf{f}$ and extracellular acidification rate (ECAR) was measured as shown in $\mathbf{~ g . ~} \mathbf{h}$, i Alveolar macrophages were harvested at 8 weeks post treatment and infected with Mtb in vitro. $12 \mathrm{~h}$ post infection mRNA expression and secretion of $\mathbf{h}$ TNF-alpha and $\mathbf{i}$ IL-1 $\beta$ as determined by qRT-PCR and ELISA, respectively. $\mathbf{j}$, $\mathbf{k}$ Alveolar macrophages were harvested from naive mice and incubated with RIF or INH/PYZ for $2 \mathrm{~h}$. Cells were washed, rested overnight, and the metabolic capacity was assessed as described for $\mathbf{e}-\mathbf{g}$. Data shown are representative of two independent experiments. Error bars represent mean \pm SEM; ${ }^{*} p<0.05,{ }^{* * *} p<0.001,{ }^{* * * *} p<0.0001 ;$ nd, not detected

used to isolate any effect on host resistance to the innate immune system. Importantly, the bacterial burden in both groups of alveolar macrophages were similar prior to adoptive transfer (Supplementary Figure 2). Three weeks after adoptive transfer, Rag1-/- mice that had received $M t b$-infected alveolar macrophages from INH/PYZ-treated mice showed a significantly higher bacterial burden in their lungs than Rag1-/- mice that received $M t b$-infected alveolar macrophages from control mice (Fig. 5f). Recently, Cohen et al. ${ }^{25}$ demonstrated that during early infection (e.g., day 8) Mtb predominantly targets alveolar macrophages. Using image analysis of infected lungs, they observed that Mtb replication occurs until day 7 post-infection. Taking this information into consideration, we examined the number of $M t b$ in alveolar macrophages directly harvested from INH/PYZ-treated mice day 6 post aerosol infection. In accordance with our previous observations, alveolar macrophages from INH/PYZ-treated mice showed significantly higher bacterial burden than alveolar macrophages from control mice (Fig. $5 \mathrm{~g}$ and Supplementary Figure 3).

\section{DISCUSSION}

As one of the most lethal infectious diseases of humankind, the discovery of antibiotics that kill Mtb provided hope that the TB epidemic would be eliminated. Indeed, the WHO has reported a $47 \%$ decrease in TB-related deaths in the past 25 years. Despite this progress, the increased susceptibility of drug-treated individuals to Mtb reinfection or reactivation brings into question whether these antibiotics may have off-target effects that prevent the generation of sterile immunity to $M t b$.

Two recent studies demonstrated that RIF treatment alone or in combination with INH and PYZ significantly decreased the diversity of the murine and human gut microbiome. ${ }^{15,16}$ In contrast to the broad-spectrum activity of RIF, treatment of mice with INH and PYZ led to more nuanced changes to the microbiome such as a decrease in certain Clostridia genera within the Firmicutes phylum that are known to have immunomodulatory effects. ${ }^{26}$ Although our $16 \mathrm{~S}$ rDNA sequencing results are consistent with these studies, the consequences of such ATTinduced dysbiosis on host resistance to Mtb infection were not known.

In the current study, we have shown that although the dysbiosis induced by INH/PYZ treatment was not extensive, it had profound effects on host resistance to Mtb infection. In sharp contrast, RIF-induced dysbiosis was drastic but with no observable effect on the anti-Mtb immune response under the conditions tested. However, we cannot exclude the possibility that in populations with unique or altered gut microbial communities that RIF may affect mechanisms of host defense. Nevertheless, our results emphasize that the functional impact of the microbiota does not always reflect the magnitude of difference between complex microbial communities, a concept supported by metagenomics studies of diverse human populations. $^{27}$

Our work also strengthens the concept of the "gut-lung axis" in host defense. Interestingly, the cell types in the lung that are affected by changes to the gut microbiota appear to differ depending on the form of immune challenge. For example, intestinal microbiota-primed alveolar macrophages showed increased reactive oxygen species-mediated killing of Klebsiella pneumoniae. ${ }^{28}$ Conversely, antibiotic-induced gut dysbiosis can promote a pro-allergic macrophage phenotype in the lung and increase susceptibility to allergic inflammation. ${ }^{29}$ In addition, treating mice with broad-spectrum antibiotics compromised the anti-viral response during influenza virus infection including decreased TNFa and IFNY production by CD $8+$ T cells. ${ }^{30}$ In the context of Mtb infection, ATT-induced dysbiosis had no discernable effect on lung-infiltrating T-cell-mediated immunity. Rather, we report an effect on the innate immune response with a particular impact on alveolar macrophages. These cells are sentinel leukocytes that constantly patrol and monitor the lower airways. Specifically, the function of alveolar macrophages in INH/ PYZ-treated mice was altered as the expression levels of $\mathrm{MHCll}$ as well as the production of TNFa and IL-1 $\beta$ were significantly reduced after $M t b$ infection. Indeed, these macrophages were more permissive for $M t b$ growth. Recent studies suggest that metabolic reprogramming of macrophages interferes with their bactericidal capacity. ${ }^{31}$ Human alveolar macrophages infected with Mtb shift their metabolism to glycolysis from oxidative phosphorylation, which is required for the production of proinflammatory cytokines (e.g., IL-1 $\beta$ ) and effective bactericidal capacity. ${ }^{32}$ These results are consistent with a recent study by Huang et al. ${ }^{33}$ demonstrating that alveolar macrophages, which rely more on fatty-acid oxidation and oxidative phosphorylation, are particularly permissive for Mtb growth. Here, we showed that alveolar macrophages from INH/PYZ-treated mice exhibited a decrease in basal respiration, maximal respiration, spare respiratory capacity, and ATP production. The spare respiratory capacity is a measure of the survival capacity of a cell and, when energy demands exceed supply, the production of ATP avoids an "ATP crisis". ATP also potently induces IL- $1 \beta$ production. ${ }^{34}$ These diminished effector functions and metabolic changes following ATT dovetail with recent results from Yao et al. ${ }^{35}$ who demonstrated that high expression of $\mathrm{MHCll}$ on alveolar macrophages represents a "defense ready" gene signature and categorized these cells as long-term alveolar macrophages. $\mathrm{MHCII}^{\text {hi }}$ alveolar macrophages showed increased glycolytic metabolism and enhanced defense mechanisms against bacterial infection. ${ }^{35}$ Collectively, these data suggest that ATT-driven changes in the microbiota promote the oxidative phosphorylation of alveolar macrophages, an intracellular environment permissive for $M t b$ growth.

At present, it is unclear how ATT-induced dysbiosis led to an altered metabolic state in alveolar macrophages and ultimately 
a

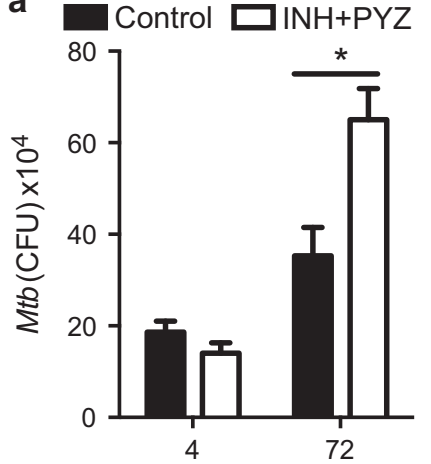

Hours after infection

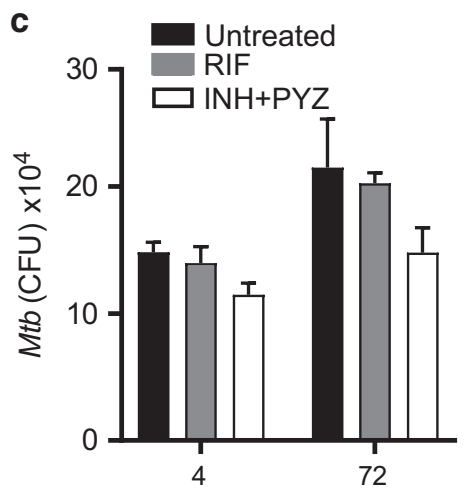

Hours after infection

e
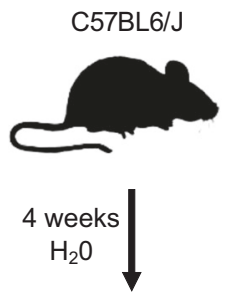

Isolate Alveolar macs

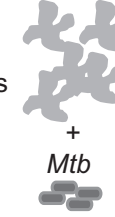

i.t. transfer infected alveolar macs

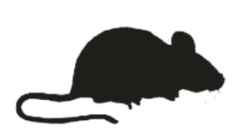

Rag1-/-

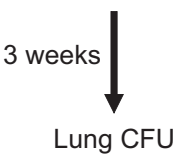

quantified b

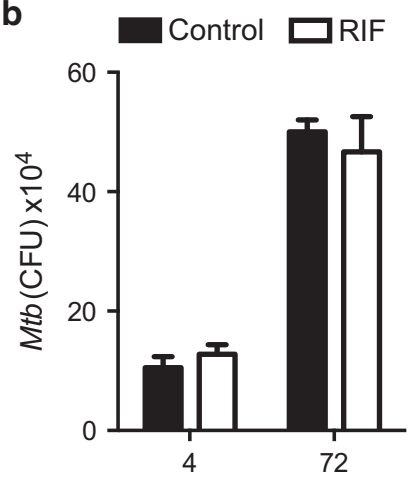

Hours after infection

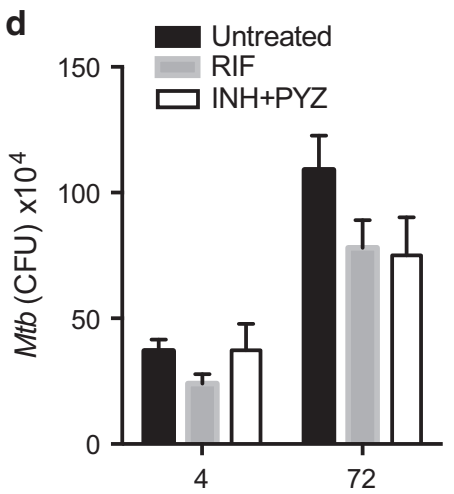

Hours after infection
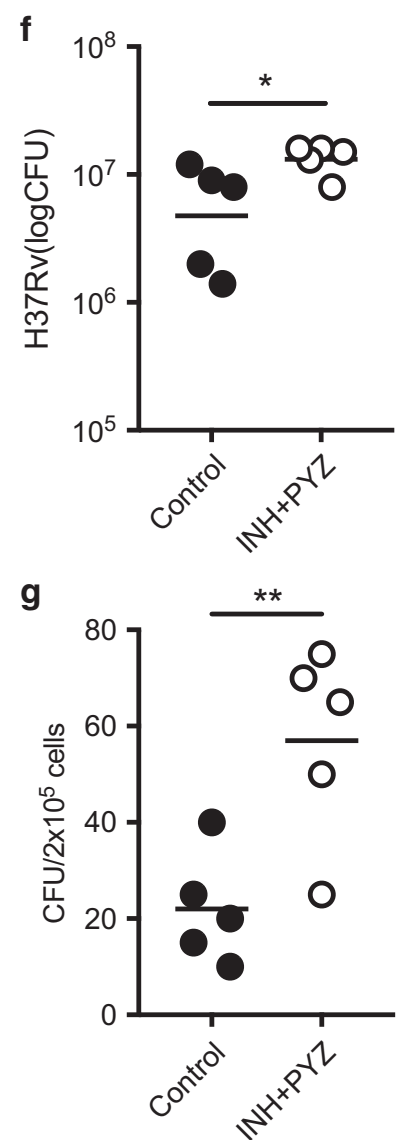
Fig. $5 \mathrm{INH} / \mathrm{PYZ}$ treatment compromises the ability of alveolar macrophages to control Mycobacterium tuberculosis growth. Mice were treated with INH/PYZ or RIF for 8 wks. Following treatment, alveolar macrophages were harvested from the BAL and bone marrow cells were differentiated into BMDM using L929-conditioned media. a, b Alveolar macrophages and c, BMDM were infected with Mtb (MOI 1) for $4 \mathrm{~h}$. Intracellular bacterial burden was assessed by plating serially diluted cell homogenates on day 3. d Bacterial load in alveolar macrophages pretreated with RIF or INH + PYZ in vitro. e Schematic representation of the alveolar macrophage transfer strategy. Alveolar macrophages were harvested from INH/PYZ-treated or control mice, infected with H37Rv (MOI 0.5) and adoptively transferred (intratracheally) into Rag1-/mice $\left(3 \times 10^{5}\right.$ cells/mouse) and lung Mtb burden was quantified 3 weeks later. $f$ Bacterial burden in the lungs ( $n=5$ per group) of Rag $1-/-$ mice 3 weeks after adoptive transfer of $M t b$-infected alveolar macrophages. $\mathbf{g} M t b$ burden in alveolar macrophages harvested from INH/PYZ or control-treated mice 6 days after aerosol infection. Data shown are pooled from two independent experiments a-d, $\mathbf{g}$ or one experiment e, f. e Error bars represent mean $\pm \mathrm{SEM} ;{ }^{*} p<0.05,{ }^{* *} p<0.01$

compromised their bactericidal activity. It is unlikely that this was owing to a direct effect of the antibiotics on macrophages as in vitro exposure of alveolar macrophages to INH/PYZ had no impact on their metabolism or their ability to kill Mtb. However, our studies do not rule out the potential impact of oral INH/PYZ treatment on the lung microbiota and certainly requires further investigation. Indeed, oral antibiotics do have the ability to alter the microbiota of the upper airways and enhance or compromise the anti-microbial functions of lung macrophages, depending on the context of immune challenge. ${ }^{36,37}$ For example, Siegel et al. demonstrated that the microbiota in newborn mice desensitizes CCL2-mediated recruitment of macrophages to the upper airways and, as a result, leaves the host more susceptible to pneumococcal infection. ${ }^{37}$ By contrast, Brown et al. ${ }^{36}$ found that the lung microbiota in adult mice enhances resistance of alveolar macrophages to pneumococcal infection by enhancing respiratory GMCSF signals and reactive oxygen species-dependent bacterial killing. These studies collectively suggest that age and microbial diversity has an important role in host resistance to infectious diseases.

An exciting, but not mutually exclusive possibility is that metabolites generated by the gut microbiota and accumulate in the blood are altered following INH/PYZ treatment and change the metabolic "set point" of the alveolar macrophage population. ${ }^{38}$ Metabolism is critical in determining the function of a macrophages in response to $M t b$. As a result of this alteration in macrophage function, $M t b$ is able to usurp the early bactericidal response and persist in the cell (depicted in Fig. 6). Consistent with this model, we observed a significant increase in the bacterial burden of alveolar macrophages at day 6 post Mtb infection, a time point in which the dominant infected cell type is the alveolar macrophage and precedes the initiation of an adaptive immune response. ${ }^{25}$ In addition, transfer of $M t b$-infected alveolar macrophages from INH/PYZ-treated mice significantly enhanced $M t b$ burden in recipient animals compared with infected macrophages from sham-treated mice. This latter result is striking given that the recipient mice harbored a population of immunocompetent alveolar macrophages that were not able to fully compensate for the defective bactericidal activity of the macrophages taken from Abx-treated mice.

Although more than two billion people are exposed to Mtb, most individuals will never develop active TB. While adaptive immunity is important for preventing Mtb dissemination and disease tolerance, ${ }^{39}$ innate immunity, pulmonary macrophages in particular, play a critical role in killing $M t b .{ }^{40}$ Thus any adverse effects on pulmonary macrophages directly compromises host resistance to TB. Although ATT is efficient in preventing TBassociated mortality, it has a limited ability to control susceptibility to reinfection or disease reactivation., 6 This limitation is compounded by the requirement for protracted use (i.e., months) of ATT and its adverse effects on health, including hepatotoxicity, and gastrointestinal dysfunction. ${ }^{4}$ Therefore, more attention should be paid to overcome the deleterious effect of ATT. Recent studies indicated that ATT-induced gut dysbiosis persists months after cessation of therapy in mice and humans. ${ }^{15,16}$ Our results suggest that specific composition of gut microbiota is essential to maintain the integrity of alveolar macrophage function against
Mtb. Our study provides a basis for novel therapeutic strategies that exploit the gut-lung axis in Mtb infection.

\section{MATERIALS AND METHODS}

Mice

Six- to 10-week-old C57BL/6 mice were procured from Jackson Laboratories. All animal studies were conducted in accordance with the guidelines of and approved by the Animal Research Ethics Board of McGill University.

Bacteria

Virulent Mtb strain $\mathrm{H} 37 \mathrm{Rv}$ was grown at $37^{\circ} \mathrm{C}$ under constant shaking in $7 \mathrm{H} 9$ medium (BD Biosciences) containing $0.2 \%$ glycerol (Fisher), 0.05\% Tween 80 (Sigma-Aldrich), and 10\% albumindextrose-catalase (BD Biosciences).

\section{Aerosol infection}

Bacteria frozen in $30 \%$ glycerol at OD- 0.5 were thawed and mixed with phosphate-buffered saline (PBS)-tween in $20 \mathrm{ml}$ for aerosol infection. Mice were infected with the Mtb strain H37Rv by the aerosol route using a nose-only exposure unit (Intox Products), which delivered $\sim 50-100$ CFU per mouse. ${ }^{41}$ CFU were determined by plating serial dilutions of lung homogenate of mice sacrificed after day 1 of infection in PBS-Tween 80 (0.05\% Tween 80$)$ on Middlebrook 7H10 medium (BD Biosciences) containing $0.5 \%$ glycerol (Fisher), $10 \%$ oleic acid-albumin-dextrose catalase (Sigma-Aldrich), and PANTA (BD Biosciences). Colonies were counted after 28 days.

\section{TB drug treatment}

Mice were treated with RIF $(10 \mathrm{mg} / \mathrm{Kg})$ or INH $(25 \mathrm{mg} / \mathrm{Kg})+\mathrm{PYZ}$ $(125 \mathrm{mg} / \mathrm{Kg})$ for 2 months supplemented in drinking water and fresh antibiotics were provided every 3-4 day. INH and PYZ are soluble in water, whereas RIF was initially solubilized in dimethyl sulphoxide in $50 \mu \mathrm{L}$ and then mixed in drinking water. Treatment was stopped 2 days prior to Mtb infection. RIF, INH, and PYZ were procured from Fisher Scientific, Sigma- Aldrich and Acros organics, respectively.

\section{Flow cytometry}

Lungs cells $\left(3 \times 10^{6}\right)$ isolated from single-cell suspension obtained from collagenase-digested lungs were first incubated with antiCD16/32 in $0.5 \%$ bovine serum albumin/PBS at $4{ }^{\circ} \mathrm{C}$ to block nonspecific $A b$ interaction with Fc receptors. For evaluation of TB10.4-specific CD8 ${ }^{+} \mathrm{T}$ cells, cells were then stained with $\mathrm{H}-2 \mathrm{~K}^{\mathrm{b}}$ TB10.4 309-318 tetramer APC and ESAT-6-PE (diluted 1:600 or 1:50, NIH Tetramer Core Facility, Emory University Vaccine Center, Atlanta, Georgia, USA), anti-CD8 (53-6.7), anti-CD19 (1D3), and anti-CD4 (RM4-5) for 30 minutes at $4{ }^{\circ} \mathrm{C}$. For innate leukocyte phenotyping, cells were stained with $\mathrm{CD} 11 \mathrm{~b}(\mathrm{M} 1 / 70), \mathrm{CD} 11 \mathrm{c}$ (N418), Siglec-F (E50-2440), F4/80 (BM8), Ly6C (HK1.4), Ly6G (1A8). For intracellular staining, Foxp3 (FJK-16s), and T-bet (4B10) antibodies were used according to the Foxp3/transcription factor staining buffer set (eBioscience) manufacturer's protocol. All antibodies were obtained from eBioscience except Siglec-F (BD Bioscience). Acquisition was done in the X20 Fortessa (BD 
Susceptibility to re-infection

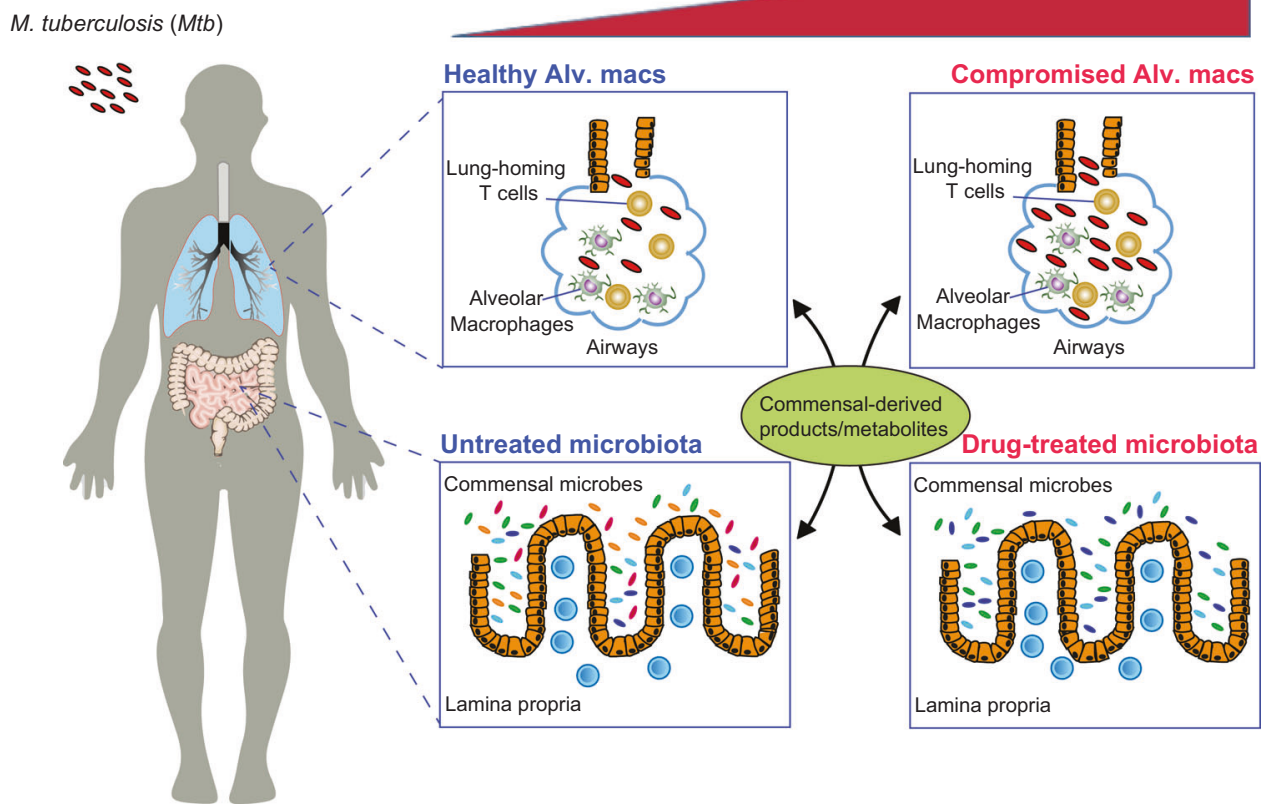

Fig. 6 Model of ATT-induced gut dysbiosis and its impact on susceptibility to Mtb infection. Gut microbiota cross talk with lung immune cells is important for their effector functions against pulmonary pathogens such as Mtb. ATT alters the gut microbiota and compromises gut-lung communication, leading to alveolar macrophage dysfunction thereby impairing early host defense mechanisms and increasing susceptibility to $M t b$ infection

Biosciences) with FACSDiva Software version 6.1.2 (BD Biosciences) and analysed with FlowJo software (TreeStar).

Isolation of alveolar macrophages

Pulmonary AM were purified from the airway of naive or treated mice by bronchoalveolar lavage (BAL) as previously described. ${ }^{42}$ In some cases, alveolar macrophages were purified from INH/PYZtreated mice day 6 post aerosol infection and seeded in 24-well plate. After $2 \mathrm{~h}$, cells were washed three times with PBS. Cells were then lysed in $250 \mu \mathrm{l}$ of water and PBS + tween 80 (0.05\%) (SigmaAldrich) for $5 \mathrm{~min}$. Mycobacterial CFU were enumerated 28 days after plating cell lysates on Middlebrook $7 \mathrm{H} 10$ agar plates incubated at $37^{\circ} \mathrm{C}$.

\section{Generation of BMDMs}

Bone marrow from both femurs and tibiae was harvested in Roswell Park Memorial Institute (RPMI) (Wisent) supplemented with $10 \%$ fetal bovine serum (FBS) (Wisent). Cells were subsequently seeded in $7 \mathrm{ml}$ RPMI supplemented with $2 \mathrm{mM} \mathrm{L-}$ glutamine, 10\% FBS, 2\% 4-(2-hydroxyethyl)-1-piperazineethanesulfonic acid, $1 \%$ non-essential amino acids, $1 \%$ essential amino acids, $0.14 \% 5 \mathrm{~N} \mathrm{NaOH}, 1 \mathrm{~mm}$ sodium pyruvate, $100 \mathrm{U} / \mathrm{ml}$ penicillin, $100 \mu \mathrm{g} / \mathrm{ml}$ streptomycin (all Wisent), and $3 \mathrm{ml}$ L929 in petridishes. After 3 days of incubation at $37^{\circ} \mathrm{C}$ and $5 \% \mathrm{CO} 2,7 \mathrm{ml}$ of supplemented RPMI and $3 \mathrm{ml}$ of L929 media. Cells were harvested at day 6 by removing the supernatant and the addition of $4 \mathrm{ml}$ cell stripper (Corning) for 20 minutes at $37^{\circ} \mathrm{C}$ and $5 \% \mathrm{CO} 2$.

In vitro infection

In total, $1 \times 10^{6}$ cells (BMDMs or alveolar macrophages (AM)) from treated or untreated mice were seeded in supplemented RPMI without penicillin/streptomycin in six-well plates, and allowed to settle overnight at $37^{\circ} \mathrm{C}$ and $5 \% \mathrm{CO}_{2}$. To assess the direct effect of antibiotics on alveolar macrophages, cells from naive mice were treated with RIF $(0.2 \mu \mathrm{g} / \mathrm{ml})$ or INH/PYZ (INH $0.5 \mu \mathrm{g} / \mathrm{ml}$; PYZ $(10 \mu \mathrm{g} /$ $\mathrm{ml})^{43}$ for $2 \mathrm{~h}$ prior to overnight incubation. The next day, cells were infected with virulent $\mathrm{H} 37 \mathrm{Rv}$ at an MOI of 1 for 4 or $72 \mathrm{~h}$ at $37^{\circ} \mathrm{C}$ and $5 \% \mathrm{CO}_{2}$. For $\mathrm{CFU}$ enumeration, cells were washed extensively with sterilized PBS and suspended in RPMI and incubated for 3 days. Cells were subsequently lysed in water and PBS + tween $80(0.05 \%)$ (Sigma-Aldrich) for $5 \mathrm{~min}$. Mycobacterial CFU were enumerated 28 days after plating cell lysates on Middlebrook $7 \mathrm{H} 10$ agar plates incubated at $37^{\circ} \mathrm{C}$.

Fecal transplant

Fecal samples were collected from five unmanipulated, co-housed mice (two pellets/mouse) and immediately pooled into a conical tube containing $2.5 \mathrm{ml}$ of cold PBS. Tubes containing feces were immediately vortexed and, after a brief centrifugation, supernatants $(2 \mathrm{ml})$ were collected and delivered to experimental mice by gavage ( $200 \mu \mathrm{l} /$ mouse) one day prior to infection and at days 1 , 2,3 , and 5 post infection.

Adoptive transfer of alveolar macrophages C57BL/6J mice were administered normal or INH/PYZ supplemented drinking water. After 4 weeks, alveolar macrophages purified from the airway of control or treated mice by BAL were infected with $\mathrm{H} 37 \mathrm{Rv}\left(\mathrm{MOI}\right.$ of 0.5 ) for 30 minutes at $37^{\circ} \mathrm{C}$ and $5 \% \mathrm{CO}_{2}$ with frequent agitation. Free bacteria were then removed by washing $5 \times$ with cold RPMI, each followed by centrifugation (1500 RPMI) for $10 \mathrm{~min}$ at $4^{\circ} \mathrm{C}$. Alveolar macrophages $\left(0.3 \times 10^{6}\right.$ cells $)$ were then resuspended in $40 \mu \mathrm{PBS}$ and then intratracheally transferred into naive Rag1-/- mice. ${ }^{4,45}$ The bacterial load prior to transfer was assessed by plating H37Rv infected alveolar macrophages on 7H10 agar plates.

qRT-PCR

DNA was isolated from fecal samples using the Power fecal DNA kit (MO Bio), according to manufacturer's instructions. DNA was quantified using a NanoDrop spectrophotometer. A260/A280 ratio of all samples was in the range of 1.90-2.00. The final reaction of RT-qPCR was performed in a volume of $10 \mu \mathrm{l}$, consisting of $1 \times$ SYBR green, $0.2 \mu \mathrm{m}$ forward primer, $0.2 \mu \mathrm{m}$ reverse primer and $5-10 \mathrm{ng}$ of CDNA. Reactions were performed at $95^{\circ} \mathrm{C}$ for $15 \mathrm{~s}$ and 
$60^{\circ} \mathrm{C}$ for 1 min for 40 cycles in Applied Biosystems step one PCR (Waltham, MA). PCR program was set according to the manufacture instructions. Analysis was done by comparative $\mathrm{Ct}$ method, where $\mathrm{Ct}$ values were normalized against a universal control.

\section{ELISA}

Alveolar macrophages harvested from INH/PYZ-treated mice were infected with $M t b$ as discussed above. After $72 \mathrm{~h}, \mathrm{IL}-1-\beta$ and TNF- $a$ was measured in culture supernatants ( $\& \& D$ systems) as per manufacturer's instruction.

16s rRNA amplification, sequencing, and analysis Feces were collected at different time point during antibiotic treatment including untreated mice as control. DNA was extracted using the Power soil DNA isolation Kit (MO Bio). PCR amplification of the V3 region of the $16 \mathrm{~S}$ rRNA gene was performed and purified PCR products were sequenced using the Illumina MiSeq platform at the McMaster Genome Facility (Hamilton, ON, Canada). ${ }^{46}$ The Microbiome Analyst web application ${ }^{47}$ was used to perform community diversity profiling and statistical analysis.

Bioenergetic Flux analyser (Seahorse) assay

Alveolar macrophages harvested from INH/PYZ-treated mice were seeded in 96-well plates from Seahorse Biosciences (Agilent Technologies). Cells were plated at 100,000 cells/well and incubated overnight at 37 degrees to minimize the effect of antibiotic treatment. Alveolar macrophages harvested from naive mice were treated with INH/PYZ (INH $0.5 \mu \mathrm{g} / \mathrm{ml}$; PYZ $(10 \mu \mathrm{g} / \mathrm{ml})$ for $2 \mathrm{~h}$. Cells were then washed and incubated overnight at $37 \mathrm{deg}$. Following resuspension in Seahorse media, the Seahorse Bioenergetic flux analyser (Agilent) was used to measure the ECAR and the OCR. Mitochondrial inhibitors were added every 10 minutes in the following order: oligomycin A $(1.5 \mu \mathrm{M})$, FCCP $(2 \mu \mathrm{M})$, and Antimycin A $(2 \mu \mathrm{M})$.

Statistical analysis

Statistical analysis for bacterial burden and leukocyte analyses were performed using an unpaired Student's $t$ test or Mann-Whitney test. One-way analysis of variance was used for group analysis using Graph Pad Prism 7 Software. For microbiome analysis, Mann-Whitney and ANOSIM were used to measure statistically significant differences in alpha and beta diversity, respectively.

\section{ACKNOWLEDGEMENTS}

We thank the Surette laboratory at McMaster University for 16S rDNA sequencing and helpful discussions related to the project. This work was supported by a CIHR operating grant (MOP-130579) to I.L.K., a ClHR Foundation Grant (FDN-143273) to M. D., the generous support of J.T. Costello Memorial Research Fund, the Richard and Edith Strauss Canada Foundation, the Lloyd Carr-Harris Foundation, and Microbiome and Disease Tolerance Centre at McGill University. N.K. is a recipient of a Fonds de recherche du Québec-Santé (FRQS) Postdoctoral Fellowship. J.X. is a Canada Research Chair in Bioinformatics and Big Data Analytics, I.L.K. is a Canada Research Chair in Barrier Immunity and M.D. holds an FRQS Award and the Strauss Chair in Respiratory Diseases.

\section{AUTHOR CONTRIBUTIONS}

N.K. performed experiments, analyzed data, and wrote the manuscript; L.M. and G.F. performed experiments and provided technical input; A.D. performed microbiome analyses and generated figures; J.X. provided technical input and data interpretation regarding the microbiome analyses; D.M. provided financial support and conceptual input; M.D. and I.L.K. conceived the original idea, supervised the project, and wrote the manuscript.

\section{ADDITIONAL INFORMATION}

The online version of this article (https://doi.org/10.1038/s41385-019-0147-3) contains supplementary material, which is available to authorized users.

Competing interests:: The authors declare no conflicts of interest.

Publisher's note: Springer Nature remains neutral with regard to jurisdictional claims in published maps and institutional affiliations.

\section{REFERENCES}

1. Cobat, A. et al. Two loci control tuberculin skin test reactivity in an area hyperendemic for tuberculosis. J. Exp. Med. 206, 2583-2591 (2009).

2. Morrison, J., Pai, M. \& Hopewell, P. C. Tuberculosis and latent tuberculosis infection in close contacts of people with pulmonary tuberculosis in low-income and middle-income countries: a systematic review and meta-analysis. Lancet Infect. Dis. 8, 359-368 (2008).

3. Menzies, D. et al. Four months of rifampin or nine months of isoniazid for latent tuberculosis in adults. N. Eng. J. Med. 379, 440-453 (2018).

4. Yew, W. W. \& Leung, C. C. Antituberculosis drugs and hepatotoxicity. Respirology 11, 699-707 (2006)

5. Pai, M. et al. Tuberculosis. Nat. Rev. Dis. Prim. 2, 16076 (2016).

6. Cox, H. et al. Tuberculosis recurrence and mortality after successful treatment: impact of drug resistance. PLoS. Med. 3, e384 (2006).

7. Verver, S. et al. Rate of reinfection tuberculosis after successful treatment is higher than rate of new tuberculosis. Am. J. Respir. Crit. Care. Med. 171, 1430-1435 (2005).

8. Becattini, S., Taur, Y. \& Pamer, E. G. Antibiotic-induced changes in the intestinal microbiota and disease. Trends Mol. Med. 22, 458-478 (2016).

9. Becattini, S. et al. Commensal microbes provide first line defense against Listeria monocytogenes infection. J. Exp. Med. 214, 1973-1989 (2017).

10. Khosravi, A. et al. Gut microbiota promote hematopoiesis to control bacterial infection. Cell Host. Microbe 15, 374-381 (2014).

11. Sekirov, I. et al. Antibiotic-induced perturbations of the intestinal microbiota alter host susceptibility to enteric infection. Infect. Immun. 76, 4726-4736 (2008).

12. Abt, M. C. et al. Commensal bacteria calibrate the activation threshold of innate antiviral immunity. Immunity 37, 158-170 (2012).

13. Khan, N. et al. Alteration in the gut microbiota provokes susceptibility to tuberculosis. Front. Immunol. 7, 529 (2016).

14. Rastogi, N. \& David, H. L. Mode of action of antituberculous drugs and mechanisms of drug resistance in Mycobacterium tuberculosis. Res. Microbiol. 144, 133-143 (1993)

15. Namasivayam, S. et al. Longitudinal profiling reveals a persistent intestinal dysbiosis triggered by conventional anti-tuberculosis therapy. Microbiome 5, 71 (2017).

16. Wipperman, M. F. et al. Antibiotic treatment for Tuberculosis induces a profound dysbiosis of the microbiome that persists long after therapy is completed. Sci. Rep. 7, 10767 (2017)

17. Huber, W. et al. Orchestrating high-throughput genomic analysis with bioconductor. Nat. Methods 12, 115-121 (2015).

18. Lyadova, I. V. \& Panteleev, A. V. Th1 and Th17 cells in tuberculosis: protection, pathology, and biomarkers. Mediat. Inflamm. 2015, 854507 (2015).

19. Shafiani, S., Tucker-Heard, G., Kariyone, A., Takatsu, K. \& Urdahl, K. B. Pathogenspecific regulatory $T$ cells delay the arrival of effector $T$ cells in the lung during early tuberculosis. J. Exp. Med. 207, 1409-1420 (2010).

20. Hrncir, T., Stepankova, R., Kozakova, H., Hudcovic, T. \& Tlaskalova-Hogenova, H. Gut microbiota and lipopolysaccharide content of the diet influence development of regulatory T cells: studies in germ-free mice. BMC Immunol. 9, 65 (2008).

21. Zhou, R., Yazdi, A. S., Menu, P. \& Tschopp, J. A role for mitochondria in NLRP3 inflammasome activation. Nature 469, 221-225 (2011).

22. Kalghatgi, S. et al. Bactericidal antibiotics induce mitochondrial dysfunction and oxidative damage in Mammalian cells. Sci. Transl. Med 5, 192ra185 (2013).

23. Shi, C. \& Pamer, E. G. Monocyte recruitment during infection and inflammation. Nat. Rev. Immunol. 11, 762-774 (2011).

24. Skold, M. \& Behar, S. M. Tuberculosis triggers a tissue-dependent program of differentiation and acquisition of effector functions by circulating monocytes. J. Immunol. 181, 6349-6360 (2008).

25. Cohen, S. B. et al. Alveolar macrophages provide an early mycobacterium tuberculosis niche and initiate dissemination. Cell Host. Microbe 24, 439-446 e434 (2018).

26. Atarashi, K. et al. Treg induction by a rationally selected mixture of Clostridia strains from the human microbiota. Nature 500, 232-236 (2013).

27. Human Microbiome Project, C. Structure, function and diversity of the healthy human microbiome. Nature 486, 207-214 (2012). 
28. Clarke, T. B. Early innate immunity to bacterial infection in the lung is regulated systemically by the commensal microbiota via nod-like receptor ligands. Infect. Immun. 82, 4596-4606 (2014).

29. Kim, Y. G. et al. Gut dysbiosis promotes M2 macrophage polarization and allergic airway inflammation via fungi-induced PGE(2). Cell Host. Microbe 15, 95-102 (2014).

30. Thaiss, C. A., Zmora, N., Levy, M. \& Elinav, E. The microbiome and innate immunity. Nature 535, 65-74 (2016).

31. Hall, C. J. et al. Immunoresponsive gene 1 augments bactericidal activity of macrophage-lineage cells by regulating beta-oxidation-dependent mitochondrial ROS production. Cell Metab. 18, 265-278 (2013).

32. Gleeson, L. E. et al. Cutting edge: mycobacterium tuberculosis induces aerobic glycolysis in human alveolar macrophages that is required for control of intracellular bacillary replication. J. Immunol. 196, 2444-2449 (2016).

33. Huang, L., Nazarova, E. V., Tan, S., Liu, Y. \& Russell, D. G. Growth of Mycobacterium tuberculosis in vivo segregates with host macrophage metabolism and ontogeny. J. Exp. Med. 215, 1135-1152 (2018).

34. Piccini, A. et al. ATP is released by monocytes stimulated with pathogen-sensing receptor ligands and induces $\mathrm{IL}-1$ beta and $\mathrm{IL}-18$ secretion in an autocrine way. Proc. Natl. Acad. Sci. USA 105, 8067-8072 (2008).

35. Yao, Y. et al. Induction of autonomous memory alveolar macrophages requires $T$ cell help and is critical to trained Immunity. Cell 175, 1634-1650 (2018).

36. Brown, R. L., Sequeira, R. P. \& Clarke, T. B. The microbiota protects against respiratory infection via GM-CSF signaling. Nat. Commun. 8, 1512 (2017).

37. Siegel, S. J., Tamashiro, E. \& Weiser, J. N. Clearance of pneumococcal colonization in infants is delayed through altered macrophage traance of pneumococcal colonization in infants is delayed through altered macrophage trafficking. PLoS Pathog. 11, e1005004 (2015).

38. Lee, W. J. \& Hase, K. Gut microbiota-generated metabolites in animal health and disease. Nat. Chem. Biol. 10, 416-424 (2014).

39. Tzelepis, F. et al. Mitochondrial cyclophilin D regulates $T$ cell metabolic responses and disease tolerance to tuberculosis. Sci. Immunol. 3, eaar4135 (2018).

40. Divangahi, M. Are tolerance and training required to end TB? Nat. Reviews Immunol. 18, 661-663 (2018).

41. Tzelepis, F. et al. Annexin 1 regulates DC efferocytosis and cross-presentation during Mycobacterium tuberculosis infection. J. Clin. Invest. 125, 752-768 (2015)

42. Zhang, X., R. Goncalves, and D. M. Mosser. The isolation and characterization of murine macrophages. Curr. Protoc. Immunol. Chapter 14, Unit 14.1 (2008).

43. Kim, J. J. et al. Host cell autophagy activated by antibiotics is required for their effective antimycobacterial drug action. Cell Host. Microbe 11, 457-468 (2012).

44. Divangahi, M. et al. Mycobacterium tuberculosis evades macrophage defenses by inhibiting plasma membrane repair. Nat. Immunol. 10, 899-906 (2009).

45. Divangahi, M., Desjardins, D., Nunes-Alves, C., Remold, H. G. \& Behar, S. M. Eicosanoid pathways regulate adaptive immunity to Mycobacterium tuberculosis. Nat. Immunol. 11, 751-758 (2010)

46. Lau, J. T. et al. Capturing the diversity of the human gut microbiota through culture-enriched molecular profiling. Genome Med. 8, 72 (2016).

47. Dhariwal, A. et al. MicrobiomeAnalyst: a web-based tool for comprehensive statistical, visual and meta-analysis of microbiome data. Nucleic Acids Res. 3 W180-W188 (2017) 